\title{
I GRUPPI PARLAMENTARI DOPO IL 1994. FLUIDITÀ E RIAGGREGAZIONI
}

\author{
di Luca Verzichelli
}

Gruppi parlamentari e sistema partitico. Continuità e mutamento

Lo studio dei gruppi parlamentari non ha mai rivestito grande importanza in Italia, anche per la generale convergenza tra gli studiosi, politologi e giuristi, intorno alla tesi della subordinazione dei gruppi stessi rispetto ai partiti, sin dall'epoca della ricostruzione democratica del dopoguerra.

In breve, l'intera esperienza della Prima Repubblica sarebbe stata caratterizzata da un sotto-sistema parlamentare facilmente controllato dai partiti (o dalle fazioni, nel caso di unità partitiche complesse) senza che le strutture politiche interne alle camere potessero ritagliarsi una propria autonomia. È vero che l'approfondimento empirico sul grado di istituzionalizzazione dei gruppi ha dimostrato un differente peso di tali strutture nei vari partiti italiani (Gatti 1987) ed è anche vero che il ruolo politico dei gruppi stessi nel policy making parlamentare può essere valutato in modo diverso a seconda della fase storica a cui facciamo riferimento (Cotta 1989). Tuttavia, almeno guardando ai dati sulla composizione e sulla stabilità dei gruppi parlamentari italiani, non ci sono dubbi sulla stretta corrispondenza tra queste strutture e un sistema partitico cristallizzato: pochi e contenuti sono stati i cambiamenti dovuti ai riallineamenti elettorali; scarsissimi i casi di variazione durante le legislature, per lo più dovuti ad eventi politici esterni al Parlamento (scissioni, aggregazioni di più partiti, ecc.).

Invece, in un contesto di transizione, come quello vissuto oggi dal sistema italiano, i gruppi parlamentari rappresentano uno dei luoghi dove maggiormente si evidenziano le trasformazioni degli attori politici rilevanti (partiti, fazioni, leadership) e le modalità di interazione tra di essi. Nella fase di assestamento che segna il passaggio da un assetto democratico ad un altro,

RIVISTA ITALIANA DI SCIENZA POLITICA / a. XXVI, n. 2, agosto 1996 
un sistema politico può infatti trovare all'interno del suo Parlamento i segni dell'instabilità e dell'incertezza. E questo è sicuramente il caso della crisi italiana degli ultimi anni. E innegabile, infatti, che alcuni degli eventi più significativi della transizione vissuta dal sistema politico abbiano avuto importanti riflessi sul Parlamento o comunque siano stati legati a questa istituzione (come nel caso dei due referendum abrogativi che hanno avviato il processo di trasformazione in senso maggioritario del sistema elettorale). Altrettanto evidente è il disagio della classe politica, a partire dall'inizio degli anni '90, nel mantenersi all'interno delle forme tradizionali di aggregazione parlamentare, a causa della crisi (prima organizzativa poi elettorale) dei vecchi partiti e del difficile consolidamento dei nuovi ${ }^{1}$.

Un fenomeno di trasformazione che intaccasse direttamente i gruppi parlamentari era del tutto prevedibile, date le premesse del cambiamento in qualche modo prefigurate già con le elezioni del 1992, ma le dimensioni di tale fenomeno, a quattro anni dall'inizio della transizione, rivestono dimensioni largamente inaspettate. Per illustrare la dinamica della crisi italiana è allora utile tornare al mutamento dei gruppi parlamentari, vero ponte tra le strutture partitiche-societarie e le istituzioni, osservandone le vicende degli ultimi anni ed individuando i diversi fattori che possono concorrere a spiegare, con un peso diverso nei vari momenti della lunga crisi italiana, tale fenomeno.

L'analisi del cambiamento nel sistema dei gruppi parlamentari può seguire diverse prospettive. Come variabili indipendenti, la trasformazione e l'instabilità dei gruppi possono essere considerate dei fattori di rallentamento nel processo di policymaking, capaci di generare feed-back sulla stessa struttura interna del Parlamento e sulle sue logiche di formazione del consenso. Come variabile dipendente, invece, questa trasformazione può essere messa in relazione con due tipi di cause: 1) la dinamica di mutamento del sistema partitico, che determina la strutturazione dei gruppi durante i passaggi elettorali e durante le stesse legislature; 2) l'impatto delle riforme del sistema elettora-

1 Un esempio evidente di tale disagio, nella fase iniziale della transizione, è stata la nascita, nel 1992, di una coalizione trasversale di parlamentari eletti in diverse liste ma uniti dal patto per le riforme. Come si ricorderà, tali parlamentari dimostrarono la propria autonomia anche in importanti occasioni politiche non legate alle riforme istituzionali (come il rifiuto di sostenere la candidatura di Forlani al Quirinale e la scelta di Scalfaro). 
le, che possono provocare un mutamento del legame tra parlamentare e collegio, e quindi un maggiore o minore livello di autonomia del parlamentare stesso rispetto al gruppo.

In questa nota seguirò la seconda prospettiva, la più immediata nell'ottica di un tentativo di interpretazione della crisi italiana. In particolare mi concentrerò sulla dinamica di variazione del personale dei gruppi durante l'ultima fase politica, offrendo una descrizione delle dimensioni e delle caratteristiche qualitative del fenomeno. Tale analisi è finalizzata all'interpretazione di una crisi più lunga e complessa del previsto: la legislatura che avrebbe dovuto superare l'impasse in cui era caduto, fin dai primi anni '90, il sistema italiano si chiude con molti «nulla di fatto» e soprattutto con un quadro politico-parlamentare tutt'altro che definito. Rifacendosi ad un noto approccio analitico relativo alla dinamica dei regimi, il fenomeno della fluidità dei gruppi parlamentari è utilizzabile dunque come un indicatore negativo di due fattori essenziali del consolidamento del nuovo regime democratico, quali il rafforzamento organizzativo dei singoli partiti e quello complessivo del nuovo sistema partitico (Morlino 1986).

In questo ambito interpretativo, il fattore esplicativo del perdurare della crisi italiana più facilmente intuibile è la debolezza dei partiti politici (o meglio, la decadenza delle vecchie formazioni unitamente al mancato rafforzamento organizzativo delle nuove). A questo si deve aggiungere tuttavia l'impatto, ancora difficile da quantificare, di un principio nuovo di selezione della leadership, prima invocato, con la stagione referendaria, e poi formalizzato attraverso l'adozione di un sistema elettorale basato sulla scelta uninominale. La prima cosa da fare è allora quella di mettere a fuoco le dimensioni e le caratteristiche dell'instabilità parlamentare nei due anni di «transizione prolungata» della XII Legislatura, per poi passare ad una prima e provvisoria discussione circa la plausibilità delle diverse spiegazioni possibili del fenomeno.

Una breve ricostruzione della storia dei gruppi durante l'esperienza repubblicana, compiuta sulla base della letteratura ${ }^{2}$,

2 Per una ricostruzione completa relativa alle prime dieci legislature cfr. Gatti (1987). Fondamentalmente la struttura del sistema dei gruppi alla Camera è rimasta immutata dal 1953 agli anni ' 80 (con alcune eccezioni significative, come la scomparsa del gruppo monarchico dopo cinque legislature, la breve presenza del gruppo PSIUP nella $\mathrm{V}$ e nella VI, o anche alcune fasi di assenza dei gruppi PRI e PSDI per mancanza del 
mostra un altissimo livello di continuità nel sotto-sistema parlamentare, sia intesa come durability delle etichette e del peso relativo delle varie formazioni (in virtù della più generale cristallizzazione del sistema partitico), che come stabilità dei gruppi stessi durante le legislature.

Inoltre si evidenzia, sfogliando la documentazione ufficiale ${ }^{3}$, un bassissimo numero di abbandoni «individuali» dei gruppi, cioè una stabilità che non attiene soltanto all'assenza di fattori di destabilizzazione del sistema partitico, ma ad un alto livello di fedeltà della classe parlamentare ai propri organismi di affiliazione. Su questo, si può supporre, ha potuto incidere per lungo tempo anche il sistema elettorale tradizionale, sia nella versione proporzionale di lista adottata per la Camera, sia in quella uninominale con quorum elettorale al $65 \%$ e recupero proporzionale dei seggi su base regionale (Senato), che rafforzava il legame tra candidato e partito scoraggiando candidature locali senza una chiara identificazione. Fino al 1994, in sostanza, non affiorano elementi significativi di trasformazione nel rapporto tra eletto e partito parlamentare.

Una più attenta analisi della qualità degli spostamenti mette tuttavia in evidenza alcuni segnali di progressivo sfaldamento del sistema partitico a partire dalla seconda metà degli anni '80: innanzitutto la presenza di un gruppo misto più frammentato rispetto al passato ${ }^{4}$, nonostante l'aumento complessivo del numero dei gruppi. In secondo luogo il fatto che i passaggi da un gruppo all'altro siano dovuti a fenomeni diversi rispetto al pas-

numero minimo di deputati). Nel 1976 nascono i gruppi dei radicali e DP (che si alternerà con il PDUP), nel 1983 quello della Sinistra Indipendente, successivamente i Verdi (1987), la Rete e la Lega Nord (1992), tutte novità che non cancellano tuttavia le formazioni già presenti. Le cose non sono dissimili al Senato, dove ovviamente troviamo una maggiore discontinuità dovuta alla mancanza del numero minimo di rappresentanti nei partiti minori. Relativamente alle variazioni intervenute nel corso delle legislature Gatti riporta, nelle sue note, soltanto sette casi relativi alla Camera e cinque al Senato. Si tratta di variazioni collegate a note trasformazioni interne dei partiti storici (nascita del PSIUP, riunificazione del PSI nel 1969, scissione nel MSI nel 1976, ecc.), destinate tuttavia a non lasciare un segno molto importante sul sistema partitico. A queste variazioni vanno aggiunte quelle del periodo più recente che menziono più avanti nel testo.

3 Su questo aspetto, proprio per la sua entità irrisoria, non vi è traccia di analisi nella letteratura storica e politologica. Senza la pretesa di fornire una serie di dati comparabili, ho compiuto una verifica sulla documentazione relativa all'ultimo quindicennio, riscontrando pochissimi casi di dimissioni a titolo personale dai gruppi parlamentari (mai più di tre per ogni legislatura in entrambe le Camere).

4 Oltre ai «tradizionali» parlamentari del partito popolare sud-tirolese e al rappresentante valdostano, troviamo, a partire dal 1983, il ritorno degli azionisti sardi, gli esponenti della Liga Veneta (poi Liga autonomista) e della Lega Lombarda. 
sato: i pochi casi di defezione, o la nascita di nuove formazioni parlamentari, erano stati infatti solitamente il frutto di scissioni evidenti, originate da conflitti intra-partitici dibattuti a lungo e pubblicamente, prima di una decisione estrema che non metteva in discussione l'esistenza delle vecchie organizzazioni. A partire dalla fine degli anni '80, al contrario, si è assistito a vere e proprie crisi di identità (e anche di organizzazione) dei partiti stessi. Un esempio di ciò è costituito dal passaggio della corrente di Romita dal PSDI al PSI (1989), che coinvolge, oltre all'ex segretario del partito, una fetta importante del personale parlamentare, facendo da preludio alla marginalizzazione del partito social-democratico. Ancora più drammatica è la fuga dal gruppo di DP dei Verdi Arcobaleno (1989), che lasciano soltanto quattro deputati nella formazione originaria, a rappresentare un partito oramai svuotato di iniziative e di mezzi.

Ma la scissione più importante, per cogliere la ristrutturazione del sistema partitico, è quella successiva all'ultimo congresso del PCI (1991): la creazione del PDS trascina nella nuova formazione parlamentare l'intero gruppo della vecchia sinistra indipendente creando, sotto il profilo parlamentare, un primo motivo di rottura rispetto alla situazione pre-esistente. Dall'altra parte la nascita del Partito della Rifondazione Comunista raccoglie, oltre alla minoranza dal vecchio PCI contraria alla svolta di Occhetto, proprio i demoproletari superstiti, determinando così la scomparsa di un altro gruppo oramai tradizionale alla Camera. Si tratta di eventi che hanno dunque la proprietà di eliminare integralmente alcuni attori della vita parlamentare, cosa che non si era verificata in occasione di altre importanti scissioni del passato, dalla nascita del PSIUP nel 1964 a quella di Democrazia Nazionale nel 1976.

Ciò nonostante, ancora con le elezioni del 1992 gli elementi di continuità rispetto al sistema partitico tradizionale sono evidenti, soprattutto nelle aree non interessate al fenomeno leghista (Sani 1992) ed anche sul piano parlamentare le novità sono limitate. Non è un caso che, oltre alla formazione del gruppo della Lega Nord in entrambe le Camere, l'unica novità sia costituita, al Senato, dalla nascita di un gruppo Verdi-La Rete, che aggrega due formazioni non necessariamente unite da affinità ideologiche, ma molto vicine sulla dimensione vecchio-nuovo (Ricolfi 1993). I nuovi tuttavia non hanno ancora sconfitto $i$ vecchi (Cotta e Verzichelli 1996): tutto è rinviato al 27 marzo 1994, quando, in mezzo alla confusione generale, l'unica cosa 
certa è la scomparsa delle etichette tradizionali: già usciti di scena il PSI (per decimazione) 5 e la DC (per cambiamento di nome), la logica delle coalizioni impone, oltre a formazioni del tutto nuove, un violento rimescolamento tra le vecchie leadership passate dal livello dei partiti a quello dei poli (Di Virgilio 1994; Vassallo 1995).

\section{L'avvio della XII legislatura: gruppi nuovi; gruppi instabili}

Le elezioni del 1994 sanciscono dunque la fine di un sistema partitico tra i più stabili del panorama occidentale, bocciando una intera classe politica e gran parte delle formazioni partitiche fino allora sulla scena. La riaggregazione dei rappresentanti a livello parlamentare avviene con grande difficoltà, seguendo strategie differenti nei tre poli creati dalla competizione maggioritaria. Nello schieramento vincente, quello di centrodestra, le forze politiche optano per il mantenimento di gruppi parlamentari separati, a dimostrazione di una maggiore autonomia delle varie formazioni ${ }^{6}$, che si trasformerà in una divisione evidente pochi mesi più tardi, con l'uscita della Lega Nord dalla maggioranza e la crisi del governo Berlusconi. Anche al centro i due attori che avevano dato vita al Patto per l'Italia mantengono una posizione autonoma: $\mathrm{i}$ deputati ed i senatori del PPI formano un proprio gruppo mentre quelli del Patto Segni aderiscono al gruppo misto dei due rami parlamentari.

A sinistra la situazione è più complessa: dopo un acceso dibattito sull'opportunità di continuare anche nella vita parlamentare l'esperienza aggregativa dei Progressist ${ }^{7}$, si perviene nelle due camere a diverse soluzioni. A Montecitorio il gruppone

5 Le vicende del gruppo parlamentare del PSI alla fine dell'XI Legislatura indicano esattamente quanto profonda fosse la crisi organizzativa dei tradizionali partiti di governo. Non si hanno scissioni, di gruppo o individuali, né dopo le dimissioni di Craxi da segretario, con l'elezione di Benvenuto, né dopo il ritiro di quest'ultimo a favore di Del Turco. Tuttavia la lotta tra le fazioni è molto forte in Parlamento: il gruppo PSI affronta tutta l'ultima fase della legislatura diviso in due tronconi, ed alla Camera si contano anche due presidenti (uno eletto dalla componente craxiana ed uno dalla opposizione interna).

$6 \mathrm{Da}$ sottolineare, in particolare, la decisione di formare un gruppo autonomo da parte dei parlamentari del CCD che pure si erano schierati insieme a Forza Italia anche nella competizione proporzionale.

7 Il dibattito è aperto da un gruppo di intellettuali e sindaci progressisti. Cfr. l'appello sul «Corriere della Sera» del 14 aprile 1994. 
Progressista-Federativo riesce a tenere insieme, oltre agli eletti del PDS, i Cristiano Sociali, i deputati della Rete ed i Verdi. Ad essi si aggiungeranno, dopo poche settimane, i rappresentanti dei due tronconi del vecchio partito socialista che hanno aderito alla coalizione (Socialisti italiani e Laburisti di Spini). Al Senato, invece, gli ex socialisti organizzano un proprio gruppo (Progressista-PSI, rinominato più tardi ${ }^{8}$ Laburista-Socialista Progressista); così pure fanno i senatori della Rete e dei Verdi (gruppo Progressisti Verdi-La Rete). Rifondazione Comunista si distacca immediatamente in entrambe le Camere, con la creazione di due gruppi autonomi, mentre la componente ex-repubblicana e laica di $\mathrm{AD}$ riesce a formare in un secondo tempo un gruppo al Senato (la Sinistra democratica) ${ }^{9}$ trovando invece rifugio alla Camera nel gruppo misto.

I tempi e le modalità della formazione dei gruppi sono insolitamente lunghi ${ }^{10}$, e ciò nonostante le indecisioni di molti parlamentari permangono per tutto il primo mese di attività parlamentare (cfr. fig. 3), segno evidente della difficoltà con cui si muove l'intera classe parlamentare nel determinare una «affiliazione». Oltre al problema della fluidità del sistema partitico si può pensare anche ad argomenti di ordine strategico, come quelli che avrebbero guidato, secondo alcuni, alla costituzione del gruppo della Sinistra democratica al Senato: l'esistenza di un gruppo autonomo, resa possibile dalle soglie permissive del regolamento della Camera alta ${ }^{11}$, avrebbe infatti garantito una so-

8 I Laburisti, già da tempo impegnati in un progetto politico diverso rispetto a quello del PSI, non aderiscono al nuovo partito che si forma immediatamente dopo le elezioni (i Socialisti italiani), da cui si dissocia anche la corrente dei Riformisti di Manca.

9 Dei dieci senatori che fondano questo gruppo, otto vengono dal misto e due dall'appena costituitosi gruppo progressista (Corasaniti e Corrao).

$10 \mathrm{La}$ vecchia legislazione proporzionale ed i limiti bassissimi per la formazione dei gruppi determinavano una procedura molto veloce per cui ogni partito rappresentato in Parlamento formava un gruppo o confluiva automaticamente in quello misto nel caso in cui il proprio numero di rappresentanti non fosse sufficiente. Nel 1994, invece, la discussione tra i progressisti circa la strategia da adottare si protrae fino all'insediamento del Parlamento (tanto è vero che la pattuglia dei socialisti si iscrive al gruppo misto per passare immediatamente al gruppo federativo).

11 Il numero minimo di parlamentari per la formazione di un gruppo è di 20 per la Camera e 10 per il Senato (art. 14 r.C e art. 14 r.S). Tuttavia entrambi i regolamenti prevedono la possibilità di formare gruppi anche meno numerosi qualora essi rappresentino partiti organizzati e presenti nei collegi elettorali in modo sufficientemente diffuso (il Senato pone in questo caso un ulteriore limite minimo di 5 membri). Sulle motivazioni di tale normativa e sull'interpretazione dei casi in cui sono stati ammessi gruppi molto limitati (piuttosto frequenti in passato) c'è stato un ampio dibattito tra gli specia- 
vra-rappresentazione di questa frangia di progressisti nelle substrutture del Senato ed anche la possibilità di partecipare alla distribuzione delle presidenze di commissione ${ }^{12}$. In generale comunque l'impressione è quella di una profonda instabilità dell'intero sistema, fin dai primi mesi della legislatura, caratterizzati dall'esperienza del governo di centro-destra ${ }^{13}$. Due fattori di discontinuità rispetto al passato sono comunque evidenti: la mancata corrispondenza «gruppo parlamentare-partito» nel settore di centro-sinistra dello spettro politico e la differenziazione, nella composizione dei gruppi, tra Camera e Senato.

Quando, nel maggio del 1994, il processo di formazione dei gruppi parlamentari si completa, con l'elezione dei rispettivi uffici di presidenza, è evidente che la transizione del sistema partitico, e più in generale dell'intero sistema politico, non è terminata. Segnali in questo senso giungono proprio dall'instabilità sul piano della struttura interna del Parlamento. In particolare si evidenzia come la transizione italiana non riesca a limitare la frammentazione del sistema partitico, come mostra l'elevato numero dei gruppi stessi. Inoltre è facilmente intuibile che il fenomeno di trasformazione dei gruppi sia destinato a continuare durante lo svolgimento della legislatura ${ }^{14}$.

\section{Le trasformazioni durante la legislatura}

Osserviamo ora invece i cambiamenti dei gruppi parlamentari nei due anni che vanno dall'insediamento della XII legislatura repubblicana al suo scioglimento, decretato dal presidente

listi. Cfr. Antonelli (1979) e Sicardi (1995). Tuttavia è evidente che il meccanismo previsto dai due articoli sopra menzionati per la tutela di piccoli ma solidi partiti è oggi del tutto insoddisfacente anche per il suo legame con il pre-esistente sistema elettorale proporzionale. Cfr. in questo senso la discussione in sede di Giunta per il regolamento alla Camera, seduta del 27 aprile 1994.

12 Aldo Corasaniti è infatti eletto presidente della I Commissione al Senato.

13 In pratica si tratta di un processo continuo. Gli spostamenti individuali iniziano pochi giorni dopo l'ufficializzazione dei gruppi: le prime defezioni (il deputato leghista Castellaneta, il senatore popolare Grillo) e le prime riaggregazioni (Melandri ed altri esponenti di AD nel gruppone progressista) risalgono al maggio 1994 (cfr. fig. 1).

14 Alcuni eventi, pur non determinando defezioni e modifiche dei gruppi parlamentari, presagiscono lo scenario di estrema fluidità che caratterizzerà l'intero svolgimento della legislatura: mi riferisco allo scontro sulla elezione del presidente del Senato (quando l'assenza di alcuni senatori popolari è decisiva per la vittoria di Scognamiglio) e alla chiamata nel governo Berlusconi del deputato pattista Tremonti. 
Scalfaro nel gennaio 1996. A tale fine presenterò, nelle pagine che seguono, una serie di dati relativi ai passaggi da un gruppo all'altro verificatisi durante tale periodo alla Camera ed al Senato $^{15}$. Nelle figure 1 e 2 ho ricostruito l'insieme complesso dei passaggi, con ulteriori informazioni relative al numero di deputati rimasti fedeli ai vari gruppi durante tutta la legislatura; a quelli acquisiti durante la legislatura stessa; a quelli usciti da ogni gruppo; e infine al numero di parlamentari rimasti in ogni formazione alla fine del periodo studiato. Per la sola Camera dei Deputati l'analisi viene approfondita con la ricostruzione grafica della distribuzione nel tempo dei 208 passaggi di gruppo che ho rilevato (fig. 3) e con una tabella che mostra l'indice di fedeltà e l'indice di acquisività di ogni gruppo parlamentare (tab. 2).

Il primo elemento da sottolineare, guardando alle due figure riassuntive, è la vivacità di molti attori politici, evidentemente non ancora riallineati in modo corretto dopo lo scossone elettorale del 27 marzo: l'urgenza di creare nuovi punti di riferimento, a livello parlamentare, è sentita soprattutto nel centro e nei settori del centro-sinistra che non vogliono un appiattimento sulle posizioni del PDS. In questa chiave possono essere letti eventi già menzionati come la nascita dei gruppi Verde-Rete e Socialisti-Laburisti al Senato, ed ancor più di quello della Sinistra democratica.

Il processo di riaggregazione dei parlamentari inizia, come ho già anticipato, praticamente all'indomani dell'apertura della legislatura ${ }^{16}$, aprendo una fase di assestamento che perdura fino all'estate del 1994 (fig. 3).

Anche durante i primi mesi del governo Berlusconi si registrano piccoli spostamenti, legati alla situazione di fermento soprattutto nella Lega Nord. Con il passare del tempo tuttavia la riaggregazione dei mini-gruppi si fa ancora più complessa e riguarda settori di parlamentari provenienti da poli diversi. I riallineamenti si concentrano nei mesi centrali della legislatura,

15 I dati utilizzati per questa analisi coprono il periodo fino al $1^{\circ}$ marzo 1996 per la Camera e fino al 22 marzo 1996 per il Senato. Ringrazio il Dott. Lippolis della Camera dei Deputati, il Dott. Felicano del Senato della Repubblica e Francesco Zucchini per la preziosa collaborazione.

16 I dati riportati nella figura 1 sono relativi alla Camera, dove riscontriamo le variazioni, già menzionate, dovute ai socialisti e agli altri progressisti, oltre ad un numero di spostamenti individuali. Ricordo tuttavia che anche al Senato il movimento dei parlamentari inizia molto presto, con la nascita della Sinistra indipendente e con il passaggio di Gianfranco Miglio al gruppo misto. 


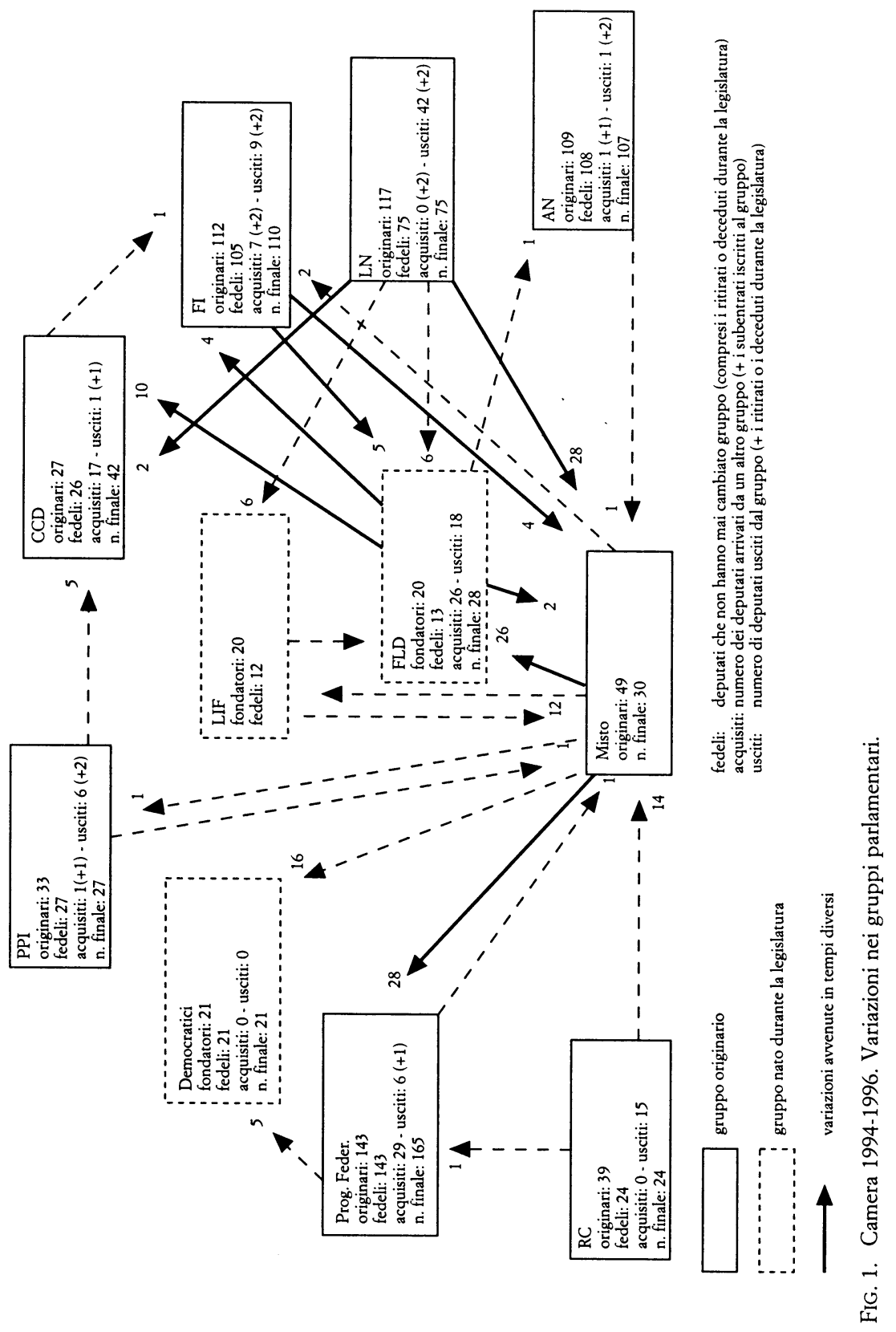




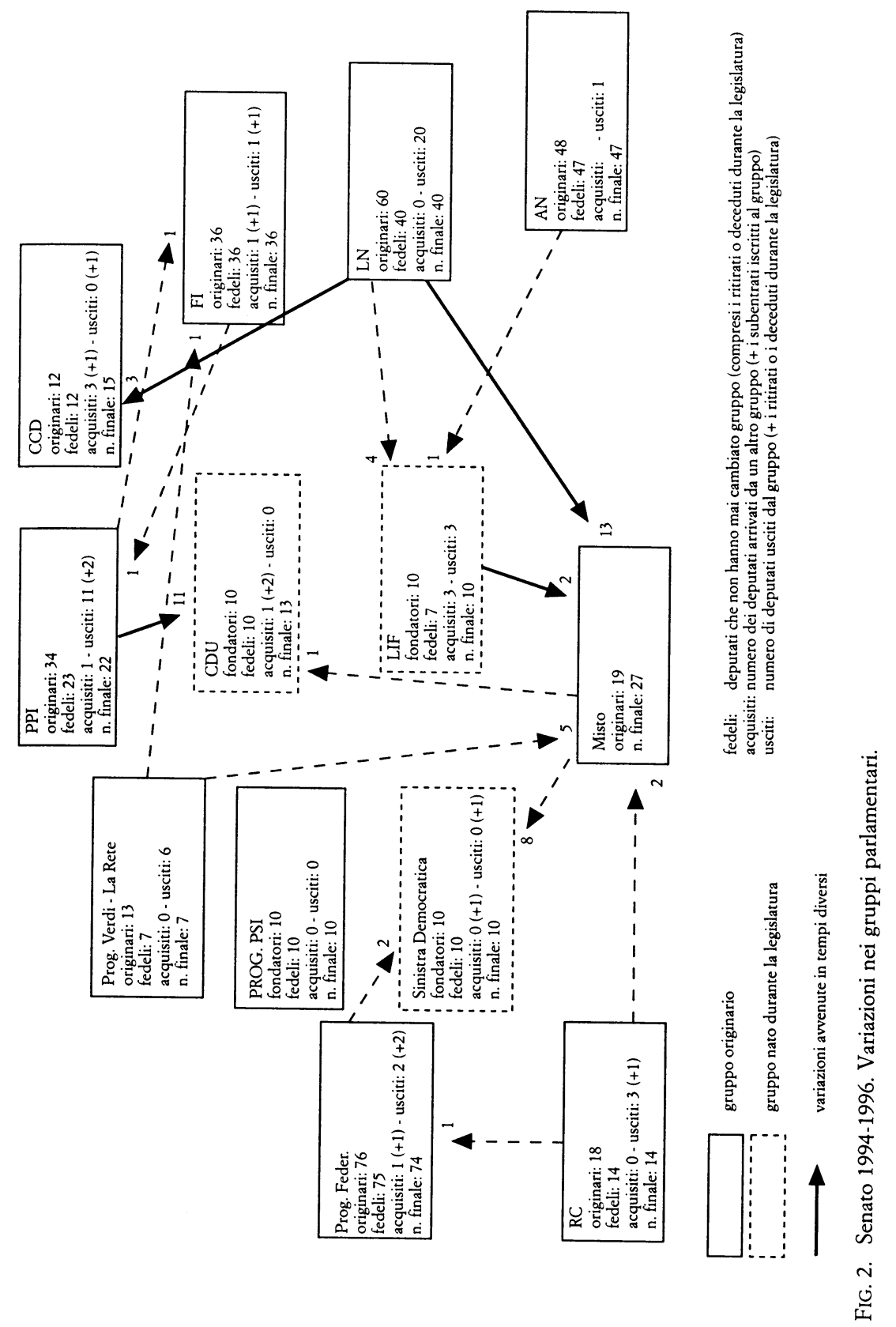




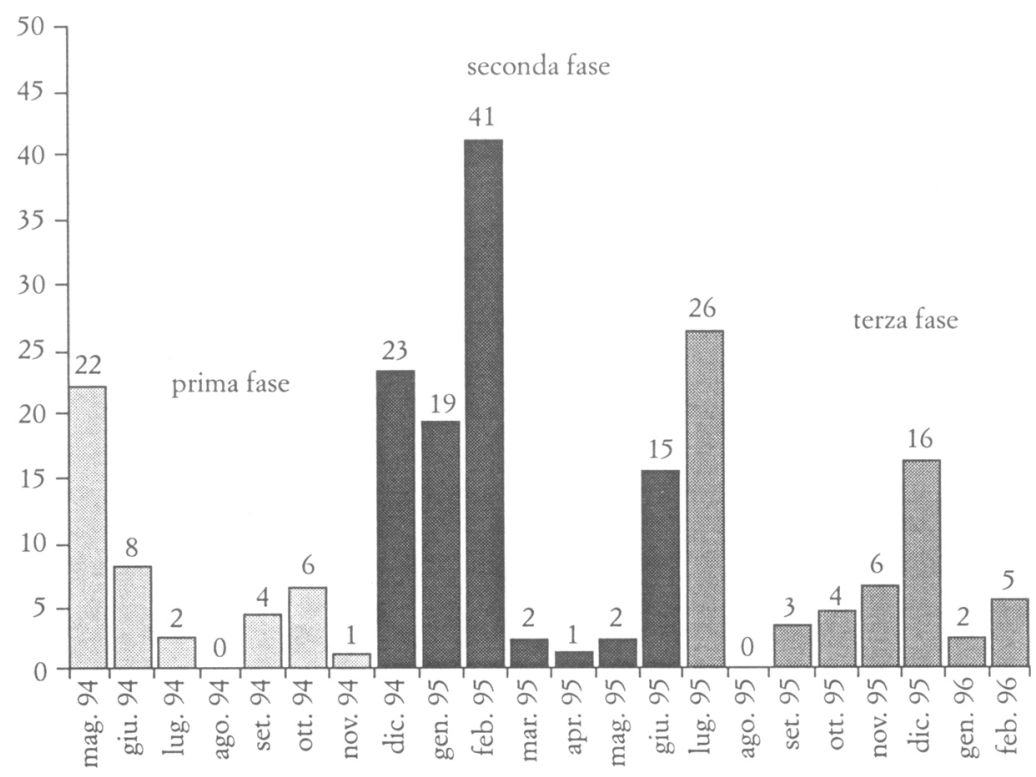

FIG. 3. Spostamenti tra i gruppi parlamentari alla Camera. 1994-1996.

quando si consumano gli eventi decisivi che incidono sugli equilibri politici e sul sistema dei partiti. La tabella 1 sintetizza tali eventi, ai quali corrispondono movimenti significativi nello scacchiere parlamentare. Due di essi (l'uscita dalla maggioranza della Lega e la divisione nel PPI) indeboliscono (senza peraltro distruggerli) due gruppi autonomi portando nel campo opposto una significativa minoranza ${ }^{17}$. Il terzo (il passaggio di Mario Segni al fronte dell'Ulivo, con la creazione della federazione dei democratici) unisce parlamentari già eletti dalla sinistra con altri (i pattisti stessi) provenienti dal polo centrale. Infine, la divisione all'interno di RC propone ai parlamentari comunisti due prospettive molto diverse: restare isolati nell'opposizione di sinistra al governo Dini o entrare nel fronte dei sostenitori di

17 La perdita nella Lega Nord è sostanzialmente la stessa nelle due camere. Molto diverso il risultato della scissione dei popolari (12 senatori e soltanto 4 deputati seguono Buttiglione nel CDU). Ciò determinerà una ulteriore differenziazione tra le due $\mathrm{Ca}$ mere: a Montecitorio i deputati usciti dal gruppo PPI saranno ospitati dal CCD, mentre al Senato si costituirà il gruppo CDU. 
TAB. 1. Fatti politici e trasformazione dei gruppi parlamentari. 1994-1995

\begin{tabular}{ll}
\hline \multicolumn{1}{c}{ Evento } & Effetti \\
\hline $\begin{array}{l}\text { Spaccatura nella Lega Nord sulla mo- } \\
\text { zione di sfiducia contro il governo Ber- } \\
\text { lusconi (dicembre 1994) }\end{array}$ & $\begin{array}{l}\text { Camera: creazione del gruppo Federalisti e } \\
\text { Liberaldemocratici (dicembre 1994) } \\
\text { Creazione del gruppo Lega Italiana Federali- } \\
\text { sta (febbraio 1995) } \\
\text { Senato: passaggio al gruppo misto dei senato- } \\
\text { ri dissidenti }\end{array}$ \\
$\begin{array}{ll}\text { Creazione di una federazione tra SI, } \\
\text { Patto Segni e AD (gennaio 1995) }\end{array}$ & $\begin{array}{l}\text { Camera: creazione del gruppo I democratici } \\
\text { (febbraio 1995) }\end{array}$ \\
$\begin{array}{l}\text { Spaccatura nel PPI sulla strategia elet- } \\
\text { torale seguita da Buttiglione (marzo }\end{array}$ & $\begin{array}{l}\text { Camera: passaggio di alcuni deputati al grup- } \\
\text { po CDD (luglio 1995) }\end{array}$ \\
$\begin{array}{ll}\text { Senato: creazione del gruppo del CDU (lu- } \\
\text { glio 1995) }\end{array}$ \\
$\begin{array}{l}\text { Spaccatura in RC sulla manovra econo- } \\
\text { mica del Governo Dini (marzo 1995) }\end{array}$ & $\begin{array}{l}\text { Camera e Senato: uscita dei Comunisti Uni- } \\
\text { tari dal gruppo di RC (giugno 1995) }\end{array}$ \\
\hline
\end{tabular}

quel governo in vista di una comune strategia elettorale con il centro-sinistra.

Lo sconvolgimento del quadro parlamentare è evidente: un numero di gruppi impensabile persino nella vecchia Italia del «proporzionale», suscettibili di verifiche e cambiamenti interni. Se da una parte infatti non stupiscono le dure perdite inflitte alle compagini parlamentari del PPI e della Lega, decimate dopo gli eventi poc'anzi menzionati, non possono non essere notati movimenti ben più ampi di parlamentari che escono anche dagli altri gruppi formatisi all'inizio della legislatura. È il caso di Forza Italia, che perde, almeno a livello di aggregazione parlamentare, la sua componente legata al vecchio PLI ${ }^{18}$. Non esitano del resto a uscire nuovamente dal gruppo ProgressistiFederativo anche i Socialisti italiani che seguono l'iniziativa di Mario Segni ${ }^{19}$.

18 Alla base del gruppo FLD, nato ancora prima della fine del governo Berlusconi, la componente leghista, sebbene maggioritaria, è autorevolmente. affiancata da un numero di deputati provenienti dal gruppo di Forza Italia, tra cui il segretario dell'Unione di Centro Raffaele Costa (che diventerà successivamente presidente del gruppo), nonché dalla componente del Patto per l'Italia che aveva sostenuto il governo di centro-destra.

19 Questo passaggio accresce ulteriormente la differenza tra Camera e Senato nella struttura dei gruppi: nel primo caso, infatti, i due segmenti più importanti del vecchio PSI (Socialisti italiani e Laburisti) saranno divisi tra Progressisti federativi e Democratici, mentre nel secondo continueranno ad essere riuniti in un unico gruppo. 
Queste trasformazioni ci ricordano quanto ancora scarsamente consolidato sia il nuovo sistema partitico italiano, e soprattutto quanto deboli siano le strutture organizzative delle singole formazioni. La difficoltà nel governare tale situazione da parte di tutti gli attori politici è naturalmente verificabile solo attraverso analisi del comportamento legislativo e del livello di disciplina interna ai gruppi. $\mathrm{Ma}$ anche indicatori più grossolani come l'instabilità della leadership di gruppo rafforzano questa impressione ${ }^{20}$.

La rivoluzione parlamentare di metà legislatura non ci riconsegna un quadro definitivo, mettendo invece in moto una spirale di ulteriori spostamenti. Non è un caso che, perlomeno alla Camera, gli effetti degli eventi appena ricordati si vedano soprattutto nei mesi di giugno e luglio 1995 (fig. 3), quando si chiude la breve esperienza del gruppo della Lega Italiana Federalista. Da questo momento, infatti, si ha la certezza che la scomparsa del sistema «tripolare» configuratosi soltanto un anno prima, non ha portato alla costituzione di un bipolarismo dai confini molto netti: il personale parlamentare rimane infatti frammentato sia nelle due ali del nuovo sistema partitico che, soprattutto, nel caotico settore centrale. La trasformazione che ne consegue investe l'universo dei gruppi ed attraversa un periodo ben più lungo rispetto alla seconda fase della figura 3 . Oltre alla fluidità nella composizione, si registrano cambiamenti significativi nei nomi, nei processi di formazione dello staff organizzativo e nelle strategie perseguite per assicurare disciplina e visibilità per la leadershi ${ }^{21}$. Perfino i gruppi parlamentari del

20 Soltanto 3 degli 8 presidenti di gruppo eletti all'inizio della legislatura hanno mantenuto la carica sino alla fine (Berlinguer, Andreatta e Brugger del gruppo misto). Per almeno tre dei presidenti sostituiti (il leghista Petrini, il forzista Della Valle e il comunista Crucianelli) il motivo del cambiamento è stato di ordine politico. Mastella (CCD) è stato sostituito dopo la sua nomina a ministro, mentre Tatarella (AN) ha preso il posto di Valensise alla fine del suo impegno ministeriale. Giova ricordare che anche i presidenti dei due gruppi creati successivamente sono cambiati: Bordon ha sostituito Mario Segni alla presidenza dei democratici, mentre Costa ha sostituito Gubetti tra i Federalisti Liberal-Democratici.

21 Oltre agli eventi ricordati nella tabella 1 , che creano fratture insanabili dentro i partiti rappresentati in Parlamento, si registrano altre situazioni che mettono in pericolo la coesione dei vari gruppi. È il caso delle votazioni circa i provvedimenti economici varati dal governo Dini, che evidenziano posizioni divergenti da parte di alcuni «cespugli» del centro-sinistra (in particolare i Verdi) o della fase precedente al voto di fiducia chiesto dal Polo in seguito al «caso Mancuso», quando sembra emergere una netta contrapposizione tra i falchi e le colombe di Forza Italia. 
partito di Fini, di gran lunga i più solidi e stabili, sono costretti a subire due piccoli ma significativi traumi come il cambiamento definitivo di nome, imposto dalla leadership ma sicuramente non accettato in modo entusiastico da tutti i componenti, e la mini-scissione, alla Camera, da parte dell'unico parlamentare riconosciutosi nelle posizioni di Pino Rauti dopo il Congresso di Fiuggi ${ }^{22}$.

Tutte le trasformazioni che ho ricordato finora sono riconducibili ad eventi ben noti, legati alla difficile strutturazione di un sistema partitico ancora oggi fortemente instabile. L'analisi dettagliata della fluidità dei gruppi mostra tuttavia un'altra caratteristica: l'estrema incertezza di ogni spostamento. La dispersione delle varie frazioni in uscita dai gruppi originari è spesso complessa e frammentata. Inoltre si verifica una molteplicità di passaggi individuali, legati spesso ad eventi isolati o personali. Per chiarire meglio cosa intendo esattamente per «incertezza» procederò ad una breve descrizione della dinamica della trasformazione dei gruppi nella XII Legislatura, relativamente alla Camera dei Deputati, dove ho rilevato un maggiore livello di fluidità: alla fine del periodo coperto dalla mia analisi (22 mesi) ho riscontrato infatti ben 208 passaggi avvenuti a Montecitorio tra gruppi parlamentari. Tali passaggi sono avvenuti in 61 momenti diversi ed hanno riguardato 122 dei 630 deputati eletti il 27 marzo $1994^{23}$.

Il grande gruppo federativo dei Progressisti mostra immediatamente una certa vitalità, arricchendosi come abbiamo visto, tra maggio e giugno 1994 , con l'entrata dei socialisti ${ }^{24}$ e di altri indipendenti transitati per il gruppo misto. Tale passaggio avviene «alla spicciolata» e non senza problemi: mentre un manipolo di esponenti di AD (sostanzialmente Bordon, Ayala e alcuni ex repubblicani) rifiutano di aderire al gruppone di sini-

22 Il cambiamento del nome avviene immediatamente dopo il Congresso di Fiuggi (7 febbraio 1995 alla Camera, 13 febbraio al Senato). In data 25 gennaio era uscito dal gruppo il deputato Della Rosa, che aveva aderito al Movimento Sociale-Fiamma Tricolore.

23 La percentuale dei fedeli rispetto ai 630 deputati eletti nel marzo 1994 è dunque del $80,6 \%$. Il numero dei passaggi è tuttavia molto più alto poiché molti parlamentari si sono spostati ripetute volte. Il record di passaggi è quello dell'ex leghista Romanello, transitato dal gruppo della Lega a quello misto, poi al nuovo gruppo LIF, quindi di nuovo al misto, a quello FLD e infine di nuovo al misto ( 5 passaggi).

24 Come ho già ricordato, i socialisti del SI aderiranno successivamente ai democratici di Segni. 
stra, altri deputati considerati in quell'area politica seguono i «cespugli» già confluiti nel grande cartello parlamentare ${ }^{25}$.

Grande incertezza anche lungo il confine tra Progressisti federati e Rifondazione Comunista: dopo l'iscrizione nel primo gruppo di alcuni eletti considerati in «quota» RC (Lanza 1995, 213) si registra anche il passaggio ai Progressisti di un altro deputato, il giornalista Giulietti, in data 20 marzo 1995. I Comunisti Unitari, ricordati nella tabella 1 , si sposteranno invece nel gruppo misto.

Anche al centro non c'è da registrare soltanto il frutto di una scissione nota (quella che porta alla creazione del CDU) ${ }^{26}$, verificandosi infatti alcuni episodi individuali che segnalano una notevole incertezza: in primo luogo la «ribellione» dei due pattisti Tremonti e Stajano all'indomani delle stesse elezioni (i due rimarranno nel gruppo misto per l'intera legislatura), e il nervosismo di alcuni deputati del $\mathrm{PPI}^{27}$.

Il settore più movimentato della Camera, nei due anni della legislatura, è stato invece quello del centro-destra ed in particolare l'area racchiusa tra Lega Nord, Forza Italia e $\mathrm{CCD}^{28}$. Le variazioni di questi gruppi sono state determinate per la maggior parte da movimenti individuali o di piccoli gruppi, e sono dunque molto difficili da ricostruire. Sinteticamente, i passaggi fondamentali di questa continua riaggregazione sono i seguenti: l'uscita di alcuni dissidenti leghisti durante la prima fase della legislatura (fig. 3) ancora prima del così detto «ribaltone» e fino alla creazione del gruppo Federalisti Liberaldemocratici (Camera), che raccoglierà molti di questi fuoriusciti insieme ai mini gruppi di Costa e Michelini; una seconda forte ondata di fuo-

25 L'ultimo, e probabilmente il più rappresentativo, sarà Ferdinando Adornato, entrato nel gruppo progressista l'8 giugno 1995.

26 Come si ricorderà la scissione si trascina tra il febbraio 1995, quando Buttiglione esprime la linea di convergenza con la politica del Polo, al mese successivo, quando il Consiglio nazionale del PPI lo sfiducia. I noti problemi di interpretazione legati proprio alla questione della Segreteria fanno scivolare molto più avanti la scissione in Parlamento (che avverrà soltanto in luglio). A Montecitorio, peraltro, soltanto 4 deputati seguono Buttiglione nel gruppo CCD.

27 In particolare c'è da ricordare il singolare movimento del deputato Lia, che staziona per qualche giorno nel gruppo misto per poi tornare tra i popolari (giugno 1994). Anche al Senato, oltre alle già menzionate astensioni di alcuni popolari in occasione del voto sulla Presidenza, c'è da registrare il passaggio del senatore Grillo al gruppo di Forza Italia.

$28 \mathrm{Nel}$ gruppo di AN non abbiamo praticamente movimenti rilevanti, a parte la già menzionata perdita del deputato legato a Rauti e l'acquisto di un ex leghista (poi gruppo misto, poi FLD) Franco Rocchetta. 
riusciti, durante la fase iniziata nel dicembre 1994, che porta alla creazione del gruppo Lega Italiana Federalista (Camera e Senato) nel febbraio 1995; l'abbandono della stessa LIF da parte di 8 deputati che passano al gruppo FLD, cosa che determina lo scioglimento del gruppo degli ex leghisti alla Camera (luglio 1995); la successiva diaspora del restante manipolo dei deputati della LIF costretti appunto dallo scioglimento di questo gruppo a tornare ancora al gruppo misto (peraltro ulteriormente alimentato da nuove defezioni avvenute nel gruppo della Lega Nord).

Questa trasformazione assume molti connotati particolari: in primo luogo, la dispersione dei «dissidenti» della Lega non è affatto omogenea. Senza tenere in conto le (moltissime) varianti di percorso individuale tra $\mathrm{i}$ gruppi, anche gli spostamenti sopra ricordati avvengono sempre con tempi dilatati e grazie ad iniziative isolate di singoli deputati o piccoli gruppi.

Se poi andiamo a vedere la destinazione di questo numeroso insieme di ex leghisti (42 unità alla Camera fino al $1^{\circ}$ marzo 1996) la situazione è ancora più frammentata e confusa: i due nuovi gruppi nati in seguito all'allontanamento di molti deputati dalla Lega Nord non catturano l'universo dei dissidenti, alcuni dei quali approdano, con i percorsi e i tempi più diversi, anche nel gruppo misto, nel CCD e in Forza Italia ${ }^{29}$.

$\mathrm{Ma}$ anche lo stesso gruppo parlamentare del movimento di Berlusconi risente dell'incertezza di alcuni suoi membri. Nei dati ufficiali che coprono il periodo sopra indicato si contano ben tre casi di uscita con successiva re-iscrizione al gruppo ${ }^{30}$, mentre in due casi (Sgarbi e Taddei) l'opzione definitiva è quella del gruppo misto. Non mancano passaggi da Forza Italia al gruppo FLD ${ }^{31}$ ed abbiamo anche il caso «estremo» di un deputato che approda addirittura al gruppo dei Progressisti ${ }^{32}$, sia pure dopo essere transitato per il gruppo misto. Alla fine della legislatura il gruppo di Forza Italia risulterà particolarmente attivo, considerando la sua estraneità ai grandi eventi elencati nel-

29 Nel marzo 1996 ancora 6 ex leghisti sono in forza al gruppo misto, 7 sono nel gruppo CCD e 4 in quello di Forza Italia. Tra questi ultimi, il fondatore e primo presidente del gruppo Federalisti e democratici, Gubetti.

30 Si tratta dei deputati Devicienti, Broglia e Mammola.

31 Quello già ricordato dei deputati UDC, e quello successivo di Tina Lagostena Bassi.

32 Si tratta dell'ex ministro Podestà, che tornerà tuttavia al gruppo misto nel finale della legislatura. 
la tabella 1 , sia sul fronte delle perdite che su quello degli «acquisti» di deputati provenienti dai gruppi vicini ${ }^{33}$.

La fedeltà del personale dei gruppi e la capacità di acquisizione di nuove forze nel corso della legislatura possono essere misurate (tab. 2) con due semplici indici che tengono conto rispettivamente del numero di deputati rimasti in quel dato gruppo durante il proprio mandato e del numero di quelli che invece sono arrivati durante la legislatura, provenienti da un altro gruppo (rapportati al numero iniziale di parlamentari).

Dalla tabella emerge che non è soltanto la Lega a dover fare i conti con un problema di tenuta della propria rappresentanza parlamentare. Tutto sommato i gruppi che sembrano essere meno colpiti da questo rischio di instabilità della classe politica sono quello progressista (che però abbiamo visto perdere i Socialisti italiani, già acquisiti nella prima parte della legislatura), il gruppo di AN e, in misura minore, quelli di CCD e FI. Vediamo inoltre che l'acquisività è molto alta nel partito più piccolo dell'alleanza di centro-destra, il CCD, che riesce ad attrarre più deputati «in fuga» di quelli raccolti complessivamente da Forza Italia e Alleanza Nazionale. Un altro gruppo caratterizzato da altissima capacità di acquisto è addirittura un movimento in fieri (sul cui futuro è certamente difficile prevedere qualcosa) come quello dei Liberal-democratici, che trovano lungo il cammino un numero di iscritti superiore a quello dei fondatori, anche se nel contempo ne perdono quasi altrettanti a favore del gruppo misto e degli altri gruppi del centro-destra.

Questa fotografia non è dissimile da quella relativa al Senato della Repubblica (cfr. fig. 2) dove, ovviamente, il minor numero di parlamentari e la stabilità dei piccoli gruppi del centrosinistra hanno rallentato i flussi durante la legislatura. Rimane tuttavia evidente, anche nella camera alta, l'elemento dell'incertezza, che determina una serie di micro-scissioni e di fughe individuali dai gruppi stessi ${ }^{34}$.

33 Oltre a pescare a più riprese nell'universo della dissidenza leghista (gruppo misto, FLD, LIF) la compagine parlamentare degli azzurri si rafforza anche grazie all'arrivo dell'ex CCD Becchetti.

34 Molti esempi potrebbero essere portati per provare tale incertezza. Mi limito a menzionare i più significativi: il protrarsi delle perdite del gruppo Lega Nord (fino alla fine della legislatura), la spaccatura nel gruppo Verdi-Rete (con l'uscita di alcuni esponenti legati al movimento di Orlando), il ritorno al gruppo misto di alcuni deputati già transitati nel gruppo LIF e il passaggio del progressista «Verde-Rete» Mancuso al gruppo di Forza Italia. 
TAB. 2. Camera dei deputati. 1994-1996. Indici di fedeltà e di acquisività dei gruppi

\begin{tabular}{lcccccc}
\hline Gruppo & $\begin{array}{c}\text { N. deputati } \\
\text { originari }\end{array}$ & $\begin{array}{c}\text { N. deputati } \\
\text { fedeli }\end{array}$ & $\begin{array}{c}\text { Indice } \\
\text { di fedeltà }\end{array}$ & $\begin{array}{c}\text { N. deputati } \\
\text { acquisiti }\end{array}$ & $\begin{array}{c}\text { Indice di } \\
\text { acquisività }\end{array}$ & $\begin{array}{c}\text { N. finale di } \\
\text { deputati }\end{array}$ \\
\hline RC & 39 & 24 & 0,63 & 0 & 0 & 24 \\
Prog. Fed. & 143 & 143 & 1 & 29 & 0,20 & 165 \\
I Democratici* & 21 & 21 & 1 & 0 & 0 & 21 \\
PPI & 33 & 27 & 0,81 & 1 & 0,03 & 27 \\
CCD & 27 & 26 & 0,96 & 17 & 0,65 & 42 \\
FI & 112 & 105 & 0,93 & 7 & 0,06 & 110 \\
LN & 117 & 75 & 0,64 & 0 & 0 & 75 \\
FLD* & 20 & 13 & 0,65 & 26 & 1,3 & 28 \\
LIF* $_{\text {AN }}$ & 20 & 12 & 0,60 & & & \\
\hline
\end{tabular}

N.B. L'indice di fedeltà è dato dal rapporto tra numero di deputati che sono rimasti nel gruppo per tutta la legislatura ed il numero del gruppo originario. L'indice di acquisività è dato dal rapporto tra numero di deputati acquisiti da un gruppo (non comprensivo dei subentranti) e consistenza finale del gruppo stesso.

* Gruppi nati durante la legislatura. Per la LIF sono considerati «fedeli» i 12 deputati che furono costretti a sciogliere il gruppo nel luglio del 1995. Ovviamente per questo gruppo non è possibile calcolare un indice di acquisività alla fine della legislatura.

\section{Conclusione}

Riepiloghiamo adesso le informazioni fin qui riportate per avviare un primo tentativo di interpretazione del mutamento parlamentare durante la XII Legislatura: abbiamo assistito al difficoltoso esordio di gruppi legati non soltanto ad un sistema partitico inedito ma anche a nuove logiche di aggregazione. Nella fase centrale della legislatura abbiamo notato come gli effetti dei mutamenti nella logica delle coalizioni post-elettorali abbiano innescato in Parlamento una catena di reazioni anche a livello individuale, proseguita fino al momento dello scioglimento delle Camere. Infine abbiamo messo in rilievo, soprattutto attraverso l'analisi degli spostamenti di gruppo alla Camera, che un importante fatto come la profonda spaccatura nella Lega Nord abbia determinato, più che una scissione, un lento processo di atomizzazione che ha coinvolto anche altri piccoli gruppi di parlamentari. La prima conclusione è dunque che la fluidità del sistema dei gruppi nella XII Legislatura del Parlamento italiano si presenta come un fenomeno eccezionale per le sue dimensioni, ma mostra, anche sotto un profilo qualitativo, alcuni caratteri patologici che ribadiscono, qualora ce ne fosse 
bisogno, le difficoltà di una transizione tutt'altro che terminata.

Gli aspetti più evidenti del fenomeno, dalle spaccature ai riallineamenti tra $\mathrm{i}$ vari gruppi, alla nascita di nuove formazioni parlamentari, alla scomparsa di altre, sono naturalmente da mettere in relazione con la dinamica del sistema dei partiti. Oggi sappiamo che gli effetti imposti dalla nuova legislazione elettorale semi-maggioritaria non sono quelli di una semplificazione del «formato» del sistema partitico (Bartolini e D’Alimonte 1995; Pasquino 1995) e che la minore polarizzazione non comporta, almeno nel breve periodo, una riduzione della complessità, a causa della persistenza di partiti intermedi la cui rilevanza viene messa in discussione (Pappalardo 1996). In ogni caso la nuova classe politica ha dimostrato un disagio generalizzato ed una indisciplina che evidenzia la precarietà della sua istituzionalizzazione: è facile prevedere, alla luce di ciò, ulteriori assestamenti sul piano del reclutamento e del ricambio nell'élite politica (Cotta e Verzichelli 1996).

$\mathrm{Ma}$, lasciando questi grandi temi a successive riflessioni, vorrei qui sottolineare un altro aspetto del fenomeno, forse più marginale per la ridefinizione della classe politica e del sistema dei partiti, ma non per questo meno rilevante: da quanto descritto sopra, emerge chiaramente che l'intero processo di riaggregazione è soltanto in parte spiegabile con gli eventi e con le date «cruciali» della legislatura, ed ha invece fatto emergere un fenomeno praticamente inedito nell'esperienza parlamentare italiana come quello dei «trasferimenti individuali». Tale fenomeno può essere messo in relazione con i due diversi fattori esplicativi menzionati all'inizio di questa nota: rispettivamente, la debolezza organizzativa dei partiti durante la fase di transizione e gli effetti della competizione maggioritaria sui comportamenti strategici dei parlamentari.

Si tratta di argomenti sui quali una più attenta riflessione, anche alla luce di nuovi dati, deve essere sviluppata. Per il momento mi limito a ribadire la plausibilità di entrambe le ipotesi, sia pure con un peso diverso nei vari settori della classe politica: i fenomeni di dissenso individuale manifestati all'interno del gruppo di Forza Italia anche sul finire della legislatura, per quanto marginali, sembrano confermare le ipotesi circa la diversità organizzativa di questo partito rispetto ad altri esempi di ieri e di oggi. Ciò può anche tradursi in rischi, limitati ma duraturi, a livello di tenuta del gruppo parlamentare.

Dall'altra parte si può sostenere che l'ostinata resistenza di 
uno «zoccolo duro» leghista, valutabile intorno al $60 \%$ del personale eletto nel 1994, testimonia la persistenza, anche sul piano organizzativo, di un partito sicuramente ridimensionato da una divisione interna molto evidente. La scelta dell'abbandono del gruppo parlamentare leghista, basata su una precisa opposizione alla linea politica di Bossi ma sicuramente anche favorita da scelte strategiche individuali ${ }^{35}$, è stata meditata a lungo e messa in atto in modo individuale da gran parte degli ex leghisti. I problemi di riaggregazione incontrati dalla minoranza uscita dal movimento di Bossi sono del resto evidenti nei dati che ho presentato, che mostrano come non sia mai stato possibile ricostruire un fronte «unico» della dissidenza ${ }^{36}$.

Ancora più complesso, infine, appare il problema per i parlamentari del centro-sinistra, per i quali si aprono strategie diverse sotto il profilo dell'aggregazione parlamentare, in attesa di un partito federativo che, per una serie di motivi, tarda a costituirsi (Vassallo 1995, 91). L'incertezza, specie tra le componenti minoritarie, provoca alcuni problemi di tenuta (probabilmente anche e soprattutto sul piano della coesione nel comportamento legislativo) sia nella versione federativa con gruppo parlamentare unico (Camera) che in quella frammentata (Senato). In particolare sembra evidente che il predominio del PDS sull'intera area elettorale genera inevitabili conflitti destinati comunque a rallentare il processo di semplificazione del quadro politico. Inoltre la presenza di una classe politica parzialmente rinnovata e sicuramente meno legata alla dirigenza partitica impone, in una fase così concitata, un rischio più alto di defezioni.

Osservando quanto è successo in tutti i settori del sistema partitico dovremo concludere che quelle del 1994 non si sono rivelate delle elezioni critiche, non avendo prodotto un riallineamento stabile a livello parlamentare. Seguendo la classica interpretazione relativa alla dinamica dei regimi politici dovremmo attenderci una fase, probabilmente ancora lunga, di transizione, per arrivare ad un più efficace controllo della leadership partiti-

35 Giova ricordare al proposito che oltre il $76 \%$ dei fuoriusciti dalla Lega alla Camera sono deputati eletti per la prima volta nel 1994 e che oltre il $90 \%$ di essi provengono da una elezione uninominale (come candidati del Polo delle Libertà, avendo contato dunque sui voti dei partiti alleati).

36 La scomparsa, alla Camera, dell'unico gruppo nato grazie al solo apporto di deputati leghisti e richiamatosi direttamente alla terminologia leghista dimostra queste difficoltà. Ancor meglio potremo valutare gli effetti della diaspora quando avremo i dati sul destino politico della dissidenza. 
ca sulla classe parlamentare. Al tempo stesso mi sembra evidente che la crisi della forma partito tradizionale e la trasformazione del ruolo partitico nelle istituzioni, fenomeni certamente non peculiari del caso italiano (Katz e Mair 1994), abbiano nel nostro paese effetti ancor più duraturi di quelli osservati nel primo biennio della transizione (ovvero la cancellazione di quasi tutte le vecchie organizzazioni partitiche). In particolare mi sembra che emerga un preciso limite, sia da parte delle formazioni politiche nuove che di quelle in qualche modo legate alla tradizione, nel tenere insieme $i$ propri rappresentanti attraverso l'istituzionalizzazione di strutture di controllo e mediazione come i gruppi parlamentari.

Relativamente al secondo fattore di disturbo della stabilità dei gruppi che indicavo all'inizio, ritengo che il fenomeno dell'abbandono individuale sarà ridotto in modo accettabile soltanto alla fine di un processo di «apprendimento» da parte degli stessi attori partitici nei confronti delle nuove logiche maggioritarie e dopo il consolidamento di una classe politica stabile e di una leadership visibile. A questo punto della transizione risulta difficile fare previsioni sulle modalità e sui tempi di un processo necessario per uscire da una sorta di emergenza permanente e per inaugurare una nuova fase politica. Tuttavia il fatto che, nonostante la fine della XII legislatura, due condizioni essenziali per la realizzazione di tale processo di apprendimento risultino ancora inattuate (una ulteriore correzione del sistema elettorale ed il consolidamento di un sistema partitico basato su un bipolarismo di alleanze o su un tendenziale bipartitismo), ci spinge a pensare che i tempi per uno scenario stabile possano essere ancora piuttosto lunghi.

\section{Riferimenti bibliografici}

Antonelli, S. (1979), I gruppi parlamentari, Firenze, La Ginestra.

Bartolini, S. e R. D'Alimonte (1995), Maggioritario ma non troppo. Le elezioni politiche del 1994, Bologna, Il Mulino.

Cotta, M. (1979), Classe politica e parlamento in Italia, Bologna, Il Mulino.

- (1989), I gruppi parlamentari tra democrazia concertata e maggioritaria, in «Biblioteca della Libertà», XXIV, n. 104, pp. 103-123.

Cotta, M. e L. Verzichelli (1996), La classe politica italiana. Cronaca di una morte annunciata? in M. Cotta e P. Isernia (a cura di), Il gi- 
gante dai piedi di argilla. La crisi del regime partitocratico in Italia, Bologna, Il Mulino.

Di Virgilio, A. (1994), Dai partiti ai poli: la politica delle alleanze, in «Rivista Italiana di Scienza Politica», XXIV, n. 3, pp. 493-547.

Gatti, C. (1987), I gruppi parlamentari nella Germania Occidentale e in Italia. Uno studio sull'istituzionalizzazione, Milano, Franco Angeli.

Katz, R. e P. Mair, (1994), How Parties Organize: Change and Adaptation in Party Organizations in Western Democracies, London, Sage.

Lanza, O. (1995), Gli eletti: il ricambio dei parlamentari, in Pasquino (1995), pp. 209-256.

Leonardi, R., R. Nanetti e G. Pasquino (1978), Institutionalization of Parliament and Parliamentarisation of Parties in Italy, in «Legislative Studies Quarterly», III, n. 1, pp. 161-186.

Morlino, L. (1986), Consolidamento democratico: definizioni e modelli, in «Rivista Italiana di Scienza Politica» XVI, n. 2, pp. 197-238.

Pappalardo, A. (1996), Dal pluralismo polarizzato al pluralismo moderato. Il Modello di Sartori e la transizione italiana, in «Rivista Italiana di Scienza Politica», XXVI, n. 1, pp. 103-145.

Pasquino, G. (1995) (a cura di), L'alternanza inattesa. Le elezioni del 27 marzo 1994 e le loro conseguenze, Soveria Mannelli, Rubbettino.

Sani, G. (1992), 1992: la destrutturazione del mercato elettorale, in «Rivista Italiana di Scienza Politica», XXII, n. 3, pp. 539-565.

Sicardi, S. (1995), Parlamento (organizzazione e funzionamento), in «Digesto delle discipline pubblicistiche», UTET.

Ricolfi, L. (1993), La geometria dello spazio elettorale in Italia, in «Rivista Italiana di Scienza Politica», XXIII, n. 3, pp. 433-474.

Vassallo, S. (1995), La politica delle coalizioni. Da un sistema partitico all'altro, in Pasquino (1995), pp. 49-97. 